\title{
Healthcare Resource Waste Associated with Patient Nonadherence and Early Discontinuation of Traditional Continuous Glucose Monitoring in Real-World Settings: A Multicountry Analysis
}

\author{
Shengsheng Yu, PhD, Biju Varughese, RPh, MBA, Zhiyi Li, MBA, MA, and Pam R. Kushner, MD, FAAFP ${ }^{3}$
}

\begin{abstract}
Background: Traditional continuous glucose monitoring (CGM) provides detailed information on glucose patterns and trends to inform daily diabetes management decisions, which is particularly beneficial for patients with a history of hypoglycemia unawareness. However, a high level of patient adherence $(\geq 70 \%)$ is required to achieve clinical benefits. The aim of this study was to assess the impact of real-world patient nonadherence and early discontinuation on healthcare resource use.

Methods: A cost calculator was designed to evaluate monthly healthcare resource waste within the first year of traditional CGM initiation by combining estimates of real-world nonadherence and early discontinuation from the literature with the wholesale acquisition costs of the current technology in the United States (for a commercial payer and for Medicare), or its equivalent in Sweden, Germany, or the Netherlands.

Results: Based on an early discontinuation rate of $27 \%$ and nonadherence rates of $13.9 \%-31.1 \%$ over the 12 months following initiation, the healthcare resource waste associated with nonadherence and early discontinuation was $\$ 220,289$ and $\$ 21,775$, respectively, for every 100 patients initiating CGM in the U.S. commercial payer scenario. In the Medicare scenario, the corresponding figures were $\$ 72,648$ and $\$ 5,675$, respectively. In both scenarios, nonadherence and early discontinuation accounted for $\sim 24 \%$ of resources being wasted within the first year of CGM initiation. Similar results were observed using the local costs in the other countries analyzed.

Conclusions: The healthcare resource waste associated with traditional CGM nonadherence and early discontinuation warrants deliberate consideration when selecting suitable patients for this technology.
\end{abstract}

Keywords: Diabetes, Continuous glucose monitoring, Adherence, Discontinuation, Cost.

\section{Introduction}

MPROVED GLYCEMIC CONTROL has been shown to reduce both the microvascular and the macrovascular complications of diabetes mellitus. ${ }^{1,2}$ Traditional continuous glucose monitoring (CGM), which involves the insertion of a small glucose sensor under the skin that measures glucose within the interstitial fluid continuously, can improve glycemic control by providing timely, actionable data about glucose levels to patients and/or providers. ${ }^{3-10}$ CGM data provide a graph of glucose levels over time, allowing trends and patterns to be seen and treatment adjusted accordingly, thus informing the management of diabetes. Better-informed treatment decisions can help patients avoid hyper- and hypoglycemia and their associated complications. Studies have shown that even patients with well-controlled diabetes can experience postprandial hyperglycemia and nocturnal hypoglycemia that are not detectable with routine self-monitoring of blood glucose; identifying these events may improve glycemic control and the patient's quality of life. ${ }^{11,12}$

\footnotetext{
${ }^{1}$ Abbott Diabetes Care, Alameda, California.

${ }^{2}$ Asclepius Analytics, New York, New York.

${ }^{3}$ Kushner Wellness Center, Los Alamitos, California.
}

(C) Shengsheng Yu, et al., 2018; Published by Mary Ann Liebert, Inc. This Open Access article is distributed under the terms of the Creative Commons Attribution Noncommercial License (http://creativecommons.org/licenses/by-nc/4.0/) which permits any noncommercial use, distribution, and reproduction in any medium, provided the original author(s) and the source are credited. 
However, the clinical benefits associated with traditional CGM are only achieved when patients demonstrate a high level of adherence over time. ${ }^{3,4,6,7,13,14}$ For example, in a large study by Battelino et al. $(n=10,501)$, patients with the highest CGM usage $(\geq 75 \%)$ had significantly lower mean blood glucose than those in all other usage groups, and were significantly more likely to achieve a hemoglobin A1c $\left(\mathrm{HbA}_{1 \mathrm{c}}\right)$ level $<8.6 \mathrm{mmol} / \mathrm{L}$ and had $50 \%$ fewer hypoglycemic episodes. ${ }^{9}$ By contrast, patients using the sensor $<25 \%$ of the time did not experience any reduction in $\mathrm{HbA}_{1 \mathrm{c}}$ relative to nonusers. Similarly, in a small study $(n=34)$, Anderson et al. found that short-term users of CGM had no statistically significant improvement in $\mathrm{HbA}_{1 \mathrm{c}}$ compared with nonusers. ${ }^{15}$

Therefore, clinical and health technology assessment guidelines, such as the Endocrinology Society and American Diabetes Association guidelines, recommend continuous use and emphasize the need for assessment of patient willingness and commitment to incorporating CGM as part of their disease management. ${ }^{16-20}$ Nonetheless, despite recent advances in CGM technology and a focus on patient education and support programs, real-world adherence and persistence rates for traditional CGM remain suboptimal. ${ }^{9,10,21}$ Studies have consistently reported that significant proportions of patients use their CGM device less often than recommended or discontinue using it at all. $3,4,9,22$

Determining the efficacy of technological interventions is complicated by the fact that data from randomized controlled trials are often not generalizable to everyday clinical practice. Such trials are performed by highly qualified and highly motivated teams of clinicians with the skill, time, and resources to support patients optimally and encourage adherence. Recently reported randomized controlled trials of traditional CGM, such as DIAMOND, HypoDE, and GOLD, have required high levels of adherence for inclusion. ${ }^{23-25}$ The difference between strict randomized controlled trial criteria and real-world clinical practice points to the likelihood that nonadherence and early discontinuation rates in real-world settings are higher than those seen in trials, potentially compromising therapeutic efficacy rates.

There is little research linking real-world CGM treatment patterns with healthcare costs. Upfront investment in CGM technology makes up a large proportion of the total first-year cost of administration. This means that a significant amount of the equipment cost is incurred before the first use. Therefore, nonadherence and early discontinuation could be associated with a substantial amount of wasted healthcare spending.

The aim of this study was to estimate the healthcare resource waste associated with traditional CGM initiation under real-world usage scenarios. First, we determined realworld CGM nonadherence and early discontinuation rates via a literature review. Second, we determined the costs of CGM. Finally, we estimated the amount of healthcare resources wasted as a result of nonadherence and early discontinuation from a healthcare payer perspective by combining the costs of CGM with the rates of nonadherence and early discontinuation in a cost calculator created for the study.

\section{Methods}

\section{Data source}

We performed a targeted literature review to identify publications reporting CGM adherence and discontinua- tion rates. The following electronic databases were searched for the period 2000 (when CGM became commercially available) to April 2017: Medline, Google Scholar, Science Direct, Web of Science, and the Cochrane Registry. The search terms were a combination of medical subject and text terms that included "diabetes mellitus"; "continuous glucose monitoring," "glucose monitoring," or "CGM"; and "adherence," "compliance," "non-adherence," "possession," "discontinuation," "usage patterns," and "treatment patterns." Additional articles were located by hand searching the references of identified publications.

The review was restricted to studies reporting real-world data (i.e., observational, database, registry, prospective, and retrospective studies) in English language publications. Our focus was on subjects $\geq 18$ years of age with type 1 or type 2 diabetes, and so, studies that only included children or adolescents were excluded. Due to the paucity of literature in this area, no other restrictions were imposed on the quality of study design. Each abstract was reviewed and the full text of each potentially relevant article assessed based on the inclusion and exclusion criteria. Existing nonadherence and early discontinuation estimates in the literature vary widely across studies, and so, the review aimed to elucidate this variance and qualitatively examine the characteristics of study samples and research designs associated with those estimates. From the identified studies, we selected the most robust and generalizable nonadherence and early discontinuation rates to enter into the cost calculator. Preference was given to the most recent data to reflect the potential impact of improvements in technology on patient behavior.

The primary outcome measures were the rates of CGM nonadherence and early discontinuation in the identified studies. Early discontinuation was defined as cessation of use within 1-year of initiation. Nonadherence was based on the proportion of days/times of CGM use after initiation and was defined as $<70 \%$ use. In other words, a patient using CGM $<70 \%$ of the time was considered nonadherent. The cutoff of $70 \%$ was chosen because this is the most common level used in the literature to date. Studies have demonstrated significantly lower $\mathrm{HbA}_{1 \mathrm{c}}$ levels in patients with $\geq 70 \%$ use versus those with $<70 \%$ use, ${ }^{6,7}$ and have also found that $\mathrm{HbA}_{1 \mathrm{c}}$ reductions are only significant in patients who use their CGM for high proportions of the time, such that when patients with lower levels of use are included in analyses, CGM is associated with no significant reduction. ${ }^{4,7}$ Thus, for the purposes of the study, partial adherence below the threshold was treated as equivalent to nonadherence, based on the premise that any additional benefit of below-threshold adherence would be minimal and unlikely to be reflected in a reduction in $\mathrm{HbA}_{1 \mathrm{c}}$. The study involved no primary data collection, and therefore, Health Insurance Portability and Accountability Act (HIPAA) and Institutional Review Board (IRB) approval were not required.

The cost of traditional CGM was based on the wholesale acquisition cost of the Dexcom G5 device. ${ }^{26-29}$ This was chosen because of its availability in all countries included in the study: United States, Germany, Sweden, and the Netherlands. In March 2017, Medicare announced that the Dexcom G5 device would be covered as durable medical equipment under Medicare part B for beneficiaries with type 1 or 2 diabetes who have been using a home blood glucose monitor and performing at least four finger-stick glucose tests per day, who are treated with insulin via multiple daily injections or an 
insulin pump, and who are receiving an insulin treatment regimen that requires frequent adjustment on the basis of therapeutic test results. ${ }^{30}$ Medicare is a U.S. governmentadministered single-payer, national social insurance program for citizens aged $\geq 65$ years, and those with disabilities and end-stage renal disease. Due to the difference between the commercial rate and the Medicare reimbursement rate, we considered two separate cost scenarios for the United States: commercial payer and Medicare. Upfront costs referred to the cost of the CGM receiver. Variable costs referred to the provider costs associated with continued use of CGM and comprised the sensor and transmitter costs.

\section{Cost calculation}

A cost calculator model was designed to evaluate healthcare resource waste within the first year of CGM initiation by combining estimates of real-world nonadherence and early discontinuation rates from the literature with cost inputs. Healthcare resource waste was defined as CGM spending that fails to produce clinically meaningful therapeutic benefit due to patient nonadherence or early discontinuation. The study period was chosen based on data availability - the majority of studies reporting nonadherence and early discontinuation rates focus on the first year after CGM initiation. Analysis was conducted using Microsoft Excel.

The cost calculator has five components. Treatment pattern inputs included estimates of CGM nonadherence and early discontinuation over the first year of use. Cost inputs included upfront and ongoing CGM-related costs. The treatment pattern processing module provided month-by-month estimates of nonadherence and early discontinuation in the first year of use. The logic within the module transforms all inputs from the literature to a uniform format that can be used in other modeling steps. The cost processing module converted raw cost inputs into month-by-month cost estimates in a uniform format. The healthcare waste module provided the raw core results, which are a function of device costs and the gap between perfect and real-world conditions. These estimates will be expressed as US\$ or the local currency in the respective country, per year per 100 CGM users.

In the model, when a patient discontinued CGM, they ceased to incur ongoing costs, and so healthcare waste was primarily traced to the unutilized portion of upfront costs. Nonadherent patients continued to incur ongoing costs, for which no meaningful clinical benefit was achieved in return, so healthcare waste was traced to both upfront and ongoing costs.

\section{Results}

\section{Literature review and cost sourcing}

Seven studies met the eligibility criteria. ${ }^{9,10,21,31-34}$ All the studies were published in the last 8 years, and the majority focused on type 1 diabetes populations. They varied in duration from 12 weeks to 2 years and encompassed a variety of designs. In 2009, Cohen et al. analyzed a prospective registry and identified 85 type 1 diabetes patients (mean age 33.2 years) starting CGM sensor-augmented pump (SAP) therapy in 6 European countries over a 12 -week period. ${ }^{21}$ In the first month, $43.4 \%$ of patients were nonadherent, and in the third month, $50.6 \%$ of patients were nonadherent. In 2013, Nørgaard et al. performed an observational study in 263 type 1 diabetes patients (mean age 28.0 years) treated with CGM SAP therapy in Europe or Israel. ${ }^{10}$ They found that the average sensor use for 12 months was $30 \%$ and that sensor use decreased with time. In a study by McQueen et al. published in 2014, 66 adult type 1 diabetes patients undergoing CGM SAP at 1 center in the United States were retrospectively identified. ${ }^{31}$ Overall, patients used CGM an average of 21 days per month ( $71 \%$ usage), with $48 \%$ of patients using it $<21$ days per month. The rate of early discontinuation was $36 \%$ over the course of 1 year. Wong et al. performed a 2014 survey within the T1D Exchange Clinic Registry, which was completed by 1,613 type 1 diabetes patients using CGM in the United States. ${ }^{33}$ Among adults, the median duration of CGM use per month was 29 days. However, the early discontinuation rate was $41 \%$ at 1 year. In a large analysis of the CareLink ${ }^{\mathrm{TM}}$ database (10,501 patients with type 1 and 2 diabetes, of whom 7,916 had used CGM over a 2-year observation period), published in 2015, Battelino et al. found that $77.8 \%$ of patients used the CGM sensor $<75 \%$ of the time. ${ }^{9}$ Among new users, the early discontinuation rate was $25.4 \%$ during the observation period and the mean time to discontinuation was 339 days. All the studies found a relationship between adherence and $\mathrm{HbA}_{1 \mathrm{c}}$ reduction, confirming the importance of maintaining high levels of adherence to benefit from CGM.

The two most recent studies, both published in 2016, were selected to provide the nonadherence and early discontinuation rates for the cost calculator. In the SENLOCOR observational study, Picard et al. evaluated 234 type 1 diabetes patients (207 adults) undergoing CGM SAP therapy in France. ${ }^{32}$ The nonadherence rate (defined as sensor wear $\leq 70 \%$ of the time) was $13.9 \%$ in months $1-3$, and $31.1 \%$ in months 4-6. We conservatively assumed that nonadherence would remain $31.1 \%$ and carried this figure forward for months 7-12. In the most recent analysis of the T1D Exchange Clinic Registry, among 1,006 adults with type 1 diabetes undergoing CGM in the United States, the early discontinuation rate was $27 \%$ over the first year. ${ }^{34}$ We used linear extrapolation to derive conservative monthly estimates of cumulative net early discontinuation.

The estimated annual cost of CGM was $\$ 9,975.36$ per patient in the United States for the commercial scenario, and \$3,237.06 for the Medicare scenario. The cost breakdown and estimated costs for the other countries analyzed are listed in Table 1.

\section{Cost calculation}

In the commercial U.S. scenario, based on the rates from the literature, the healthcare resource waste associated with nonadherence and early discontinuation in the first year was $\$ 220,289$ and $\$ 21,775$, respectively, for every 100 patients initiating CGM. Thus, nonadherence was responsible for the majority of healthcare resource waste (Fig. 1). In combination, nonadherence and early discontinuation wasted $24.3 \%$ of healthcare spending on CGM equipment within the first year of initiation (Fig. 2). In the Medicare scenario, nonadherence and early discontinuation were responsible for $\$ 72,648$ and $\$ 5,675$ of wasted healthcare resources, respectively, for every 100 patients initiating CGM. The difference in healthcare waste between the commercial and Medicare scenarios was mainly due to the lower cost of CGM within the Medicare fee schedule. Thus, in the Medicare scenario nonadherence and early discontinuation were associated with 
Table 1. Continuous Glucose Monitoring Costs by Components and by Countries

\begin{tabular}{|c|c|c|c|c|c|}
\hline & U.S. commercial plan ${ }^{\mathrm{a}}$ & U.S. Medicare ${ }^{\mathrm{a}}$ & Sweden & Germany & The Netherlands \\
\hline \multicolumn{6}{|l|}{ Individual component costs } \\
\hline Receiver/system & $\$ 793.80$ & $\$ 256.50$ & $2300 \mathrm{SEK}$ & $€ 714.00$ & $€ 578.00$ \\
\hline Transmitter ${ }^{\mathrm{b}}$ & $\$ 453.68$ & NA & $2100 \mathrm{SEK}$ & $€ 514.00$ & $€ 310.00$ \\
\hline Sensor pack ${ }^{\mathrm{c}}$ & $\$ 141.67$ & NA & $625 \mathrm{SEK}$ & $€ 74.75$ & $€ 74.50$ \\
\hline \multicolumn{6}{|c|}{ Upfront and ongoing monthly costs } \\
\hline Upfront costs & $\$ 793.80$ & $\$ 256.50$ & $2300 \mathrm{SEK}$ & $€ 714.00$ & $€ 578.00$ \\
\hline Ongoing monthly costs ${ }^{\mathrm{d}}$ & $\$ 765.13$ & $\$ 248.38$ & $3408 \mathrm{SEK}$ & $€ 495.25$ & $€ 426.17$ \\
\hline
\end{tabular}

${ }^{\mathrm{a}}$ The CGM cost for commercial plan is based on wholesale acquisition cost. The Medicare fee schedule for purchasing a new CGM monitor/receiver ranges from approximately $\$ 236$ to $\$ 277$, and there is a monthly fee schedule amount that covers sensors, transmitters, alkaline batteries used in the glucose monitors, and a blood glucose monitoring necessary for calibration of the CGM. Thus, no individual component costs are available for this scenario.

${ }^{\mathrm{b}}$ The transmitter must be replaced every 3 months.

${ }^{\mathrm{c}}$ The sensor must be replaced every 7 days. A pack covers 1 month.

${ }^{\mathrm{d}}$ Based on 4 transmitters and 12 packs of sensors per year.

CGM, continuous glucose monitoring.

a similar proportion of resources being wasted in the first year of CGM initiation, at $24.2 \%$. Comparable results and patterns were observed using the local costs in Sweden, the Netherlands, and Germany, with slightly higher rates of wastage in Germany at $25.5 \%$ (Table 2).

\section{Conclusions}

In this study, we found that CGM nonadherence and early discontinuation are likely to be responsible for significant waste of healthcare resources. Based on our calculations, almost one-quarter of healthcare spending on traditional CGM could be wasted through nonadherence and early discontinuation. These calculations may even underestimate healthcare waste. We made a conservative assumption with regard to nonadherence in months $7-12$. If the nonadherence rate actually rose during this period, so would the amount of waste. In addition, if early discontinuation occurred disproportionately in the early months of initiation rather than at a constant rate throughout the first year, as we assumed, the amount of healthcare waste would again be greater. Furthermore, patients in the SENLOCOR study were trained and followed up by a medical team, and so may have had better adherence than the average patient in real-world clinical practice. In fact, adherence was higher than that seen in some randomized trials, such as the RealTrend study, in which only $69 \%$ of patients wore the sensor $>70 \%$ of the time. ${ }^{7}$

Recent randomized controlled trials performed to evaluate the efficacy of traditional CGM, such as DIAMOND, HypoDE, and GOLD, have shown strong results for the effect of $\mathrm{CGM}$ on $\mathrm{HbA}_{1 \mathrm{c}}$ reduction. ${ }^{23-25}$ However, these trials required a high level of adherence $(>80 \%)$ as part of their inclusion and

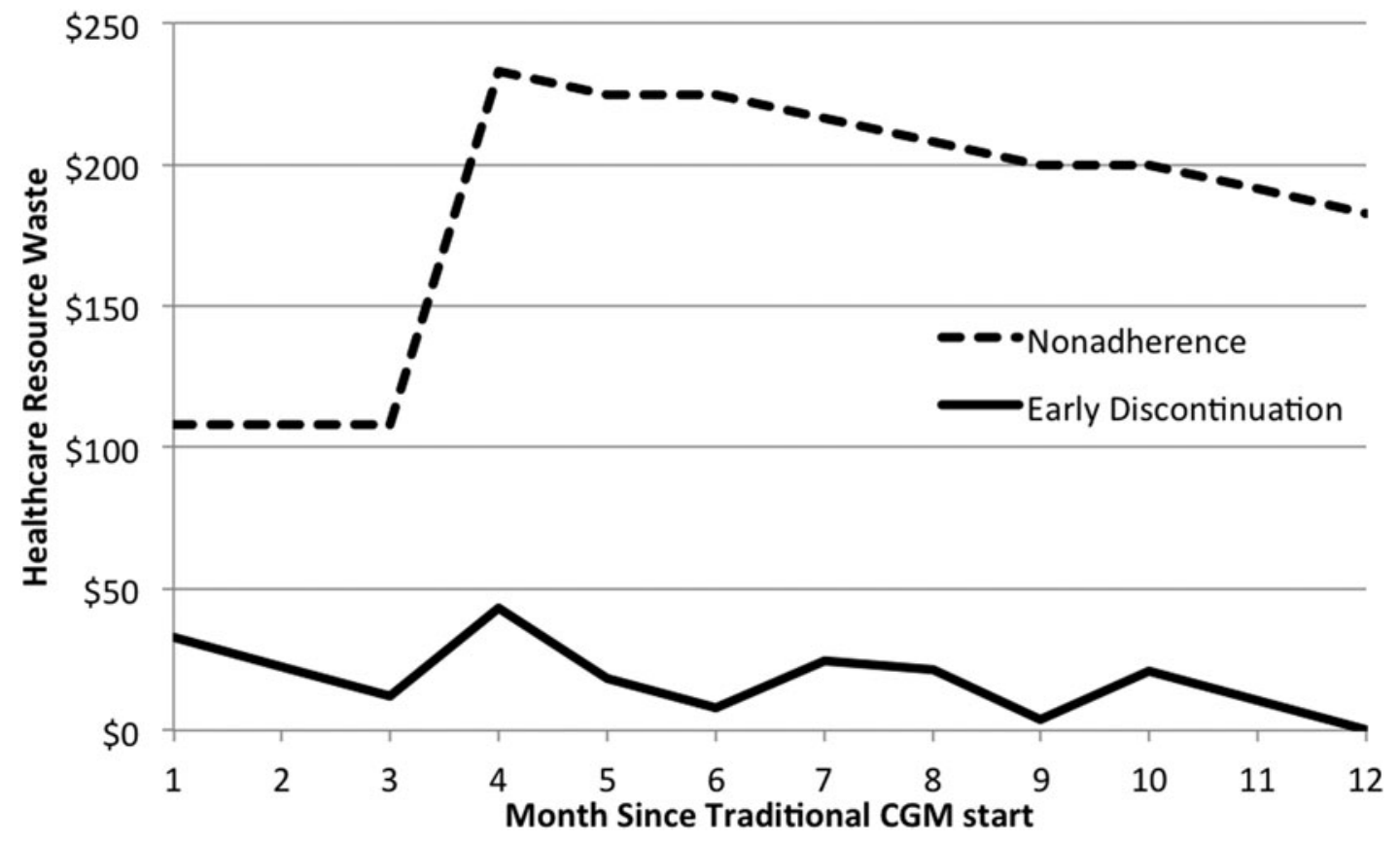

FIG. 1. Healthcare resource waste over time due to nonadherence and early discontinuation in the United States from the commercial payer perspective. 


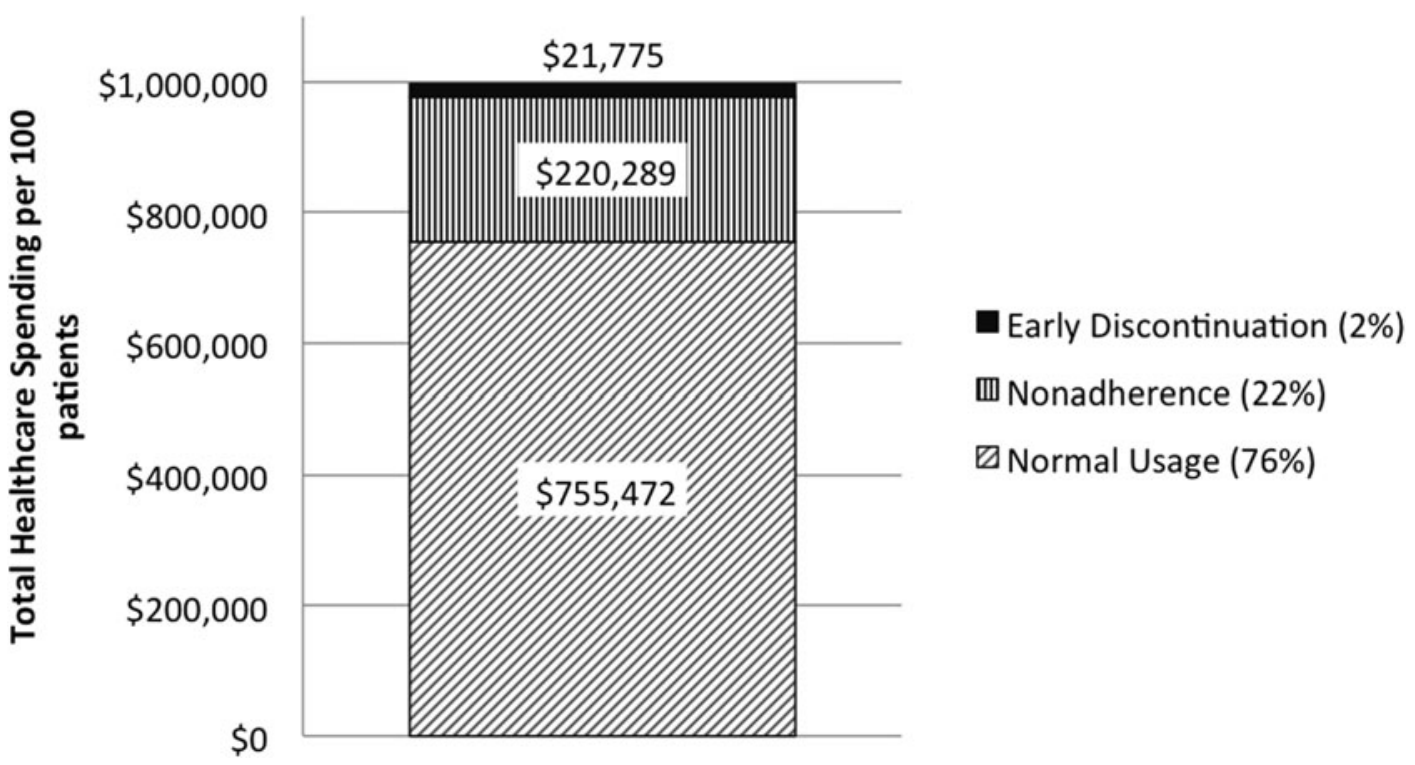

FIG. 2. CGM spending according to patient usage in the United States from the commercial payer perspective. CGM, continuous glucose monitoring.

exclusion criteria, and therefore, the results may not be generalizable to real-world CGM populations. In all the real-world studies we identified, suboptimal adherence was seen in high proportions of patients, and thus, it is likely that high proportions of patients initiating CGM in real-world settings will not achieve the results seen in randomized controlled trials.

Although the benefit of a CGM system is only achieved when patients continue to use it, much of the investment is made up-front with the expectation that the user will realize the full benefit over years with optimal adherence. Nonadherence and early discontinuation thus constitute an economic loss to the payer that cannot be recuperated. As optimal CGM use is associated with improved glycemic control and fewer hypoglycemic episodes, nonadherence and early discontinuation are also associated with what Rodbard et al. call "cost to society for failure to implement CGM." 35 Such costs include the treatment of severe hypoglycemia, hospitalizations, morbidity, and mortality, as well as costs of inadequate glycemic control for patients' quality of $\operatorname{life}^{36}$ and long-term diabetes complications.

Understanding the reasons for nonadherence and early discontinuation will be crucial for improving the rate of patient adherence and reducing healthcare resource waste. Commonly quoted reasons for early discontinuation include problems with equipment and sensor inaccuracy; intrusiveness and inconvenience; skin irritation, pain, and insertion site bleeding; too many alarms; and issues with insurance and reimbursement. ${ }^{9,37}$ Factors contributing to nonadherence are similar, but may also include inconsistent recommendations by physicians, ${ }^{21,35}$ and lower levels of patient engagement with their diabetes management, with two studies finding that a low frequency of blood glucose measurement per day before CGM initiation predicted low CGM adherence. ${ }^{9,14}$ In a recent study by Tanenbaum et al., including 1,503 patients in the T1D Exchange Clinic Registry, the most common barriers to CGM uptake were cost (61\%), the hassle of wearing the device $(47 \%)$, and disliking devices on one's body $(35 \%){ }^{22}$ Concerns about intrusiveness and alarm frequency, accuracy, and physical discomfort were also present.

Cost and reimbursement constraints have frequently been cited as factors in nonadherence and early discontinuation. ${ }^{35,37}$ However, Battelino et al. found that in countries with $100 \%$ CGM reimbursement, $32 \%$ of patients used CGM $<25 \%$ of time, indicating that reimbursement constraints may not be the primary cause of low adherence. ${ }^{9}$ de Bock et al. found that sensor accuracy was the strongest factor associated with adherence. ${ }^{38}$ In another study, Mastrototaro et al. found that many patients were setting their CGM alert thresholds at levels that produced frequent alarms, resulting in annoyance and contributing to nonadherence, and early discontinuation. ${ }^{39}$ In addition, the majority of studies have found that patients $\geq 25$ years of age have higher levels of CGM adherence and thus increased glycemic benefit compared with younger patients. ${ }^{13,14,21}$

There are few studies on how to improve patient adherence and persistence with CGM. Patient involvement appears to be

Table 2. Healthcare Resource Waste in European Countries for Every 100 Patients Initiating Continuous Glucose Monitoring

\begin{tabular}{lcccr}
\hline Country & Due to nonadherence & Due to early discontinuation & Total waste & Waste \% \\
\hline Sweden & 954,000 SEK & 84,300 SEK & $1,038,300$ SEK & 24.1 \\
Germany & $€ 147,009$ & $€ 22,446$ & $€ 169,455$ & 25.5 \\
The Netherlands & $€ 125,698$ & $€ 15,306$ & $€ 141,004$ & 24.8 \\
\hline
\end{tabular}


crucial, and this requires education and training to help patients to interpret and react to alarms and CGM data. Battelino et al. found that sensor use in the first month was significantly associated with long-term use, such that only $0.4 \%$ of patients in the highest usage group discontinued CGM during the observation period compared with $52.9 \%$ of those in the lowest usage group, and the authors speculate that patient education and training during this early period might be helpful in promoting adherence. ${ }^{9}$ A similar pattern was seen in the Juvenile Diabetes Research Foundation (JDRF) Continuous Glucose Monitoring Study, indicating an opportunity for early intervention to have long-term benefits. ${ }^{14}$

Some governments that cover CGM devices have imposed criteria for CGM use in an attempt to select for patients who are likely to be adherent. For example, the UK National Institute for Health and Care Excellence requires a 3-month trial period, after which the technology is only funded if it is effective and the patient wants to continue with it. ${ }^{19}$ In Australia, CGM requires endorsement from a healthcare practitioner, who must confirm that the patient is expected to benefit from the device and has the capability and willingness to use it. ${ }^{20}$ It must be acknowledged that patient education and assessing the readiness and compliance require staff time, and thus it increases costs. Future studies on the cost-benefit equation with regard to improving CGM compliance would be welcome.

Another factor that would be expected to improve both adherence and persistence is improvements in CGM devices as this technology evolves; for example, improved accuracy, elimination of the need for user calibration, and lower device costs. Assessment of adherence as newer devices enter the market will be important.

It has recently been argued that we are at a watershed moment for CGM where its accuracy has improved to the point where it is likely to be approved for use across the world. ${ }^{35} \mathrm{In}$ addition to the CGM coverage mentioned above in United Kingdom and Australia, healthcare systems such as Medicare and the German Federal Joint Committee (G-BA) are already starting to approve CGM for diabetes patients receiving intensive insulin therapy, meaning that global CGM usage is likely to increase considerably in the near future. ${ }^{30,40}$ However, the reimbursement criteria rarely require patient persistence or adherence.

Our study demonstrates that lack of patient persistence and adherence put scarce healthcare resource spending at risk of potential waste. Given the variability of adherence to treatments among diabetes patients and the increasing availability of CGM devices, proper evaluation of patient willingness and capability to incorporate CGM as part of their diabetes management is essential to preserving valuable healthcare resources for those who will truly benefit from the technology. Real-world patient adherence and persistence and the disconnect between them and randomized clinical trial data should be taken into consideration by healthcare providers making prescribing decisions and by payers making reimbursement decisions. Interventions to promote patient adherence will also be crucial to prevent substantial waste when CGM is approved on large scales.

\section{Limitations}

Cost calculator studies are valuable for estimating the direct and indirect costs of treatment under specific conditions modeled on real-world data. However, such studies have limitations. Cost models derive their assumptions from published literature, and therefore, one set of limitations relates to secondary analyses, such as publication bias and external validity of the original studies. It is possible that the chosen studies had patient populations that are not representative of the overall population of CGM users. Thus, the cost calculator results are limited in their generalizability by the samples of the original studies. Furthermore, individual studies differ in their estimates of core variables such as nonadherence and early discontinuation rates, and it is possible that the nonadherence and early discontinuation rates examined are not representative of all CGM populations in clinical practice.

A further limitation relates to our choice of $\geq 70 \%$ CGM use as the cutoff for adherence. There are few studies to date examining populations of patients using their CGM devices for varying proportions of time (i.e., subdividing patients using the sensor $<70 \%$ of the time into different levels of use). Therefore, we cannot be sure that patients with adherence at a level such as $50 \%-70 \%$ do not achieve similar levels of benefit to patients with adherence $\geq 70 \%$.

We chose the Dexcom G5 device to represent CGM in our study because it is currently available in all of the countries we examined, and it has also recently been approved for coverage under Medicare part B, expecting an increased usage among the elderly population for whom the real-world patient adherence might be even more challenging. However, this also means that our findings may not be generalizable to diabetes patients using other CGM devices. Our estimations may also not reflect potential differences in adherence in patients using CGM either with or without insulin pump systems. The Wong et al. T1D Exchange Clinic Registry study included multiple CGM products, as well as both pump and nonpump users. ${ }^{33}$ CGM use was significantly more likely in patients using an insulin pump in that study.

Despite these limitations, cost calculators are important as they provide more realistic expectations of resource use and costs in real-world clinical practice, rather than assuming ideal treatment conditions.

\section{Acknowledgments}

The authors thank Clare Byrne for medical writing assistance as well as Dr. Lynne H. Milgram for her compensated guidance on study design.

\section{Author Disclosure Statement}

S.Y. and B.V. are employees and stockholders of Abbott Diabetes Care. Abbott Diabetes Care provided funding to Asclepius Analytics for their work on study execution and publication preparation. Z.L. is an employee of Asclepius Analytics. P.R.K. is a compensated consultant for Asclepius Analytics and is on a scientific advisory board for Abbott Diabetes Care.

\section{References}

1. Diabetes Control and Complications Trial Research Group: The effect of intensive treatment of diabetes on the development and progression of long-term complications in 
insulin-dependent diabetes mellitus. N Engl J Med 1993; 329:977-986.

2. Gore MO, McGuire DK: The 10-year post-trial follow-up of the United Kingdom Prospective Diabetes Study (UKPDS): cardiovascular observations in context. Diab Vasc Dis Res 2009;6:53-55.

3. Deiss D, Bolinder J, Riveline JP, et al.: Improved glycemic control in poorly controlled patients with type 1 diabetes using real-time continuous glucose monitoring. Diabetes Care 2006;29:2730-2732.

4. Hirsch IB, Abelseth J, Bode BW, et al.: Sensor-augmented insulin pump therapy: results of the first randomized treatto-target study. Diabetes Technol Ther 2008;10:377-383.

5. Bailey TS, Zisser HC, Garg SK: Reduction in hemoglobin A1c with real-time continuous glucose monitoring: results from a 12-week observational study. Diabetes Technol Ther 2007;9:203-210.

6. O'Connell MA, Donath S, O'Neal DN, et al.: Glycaemic impact of patient-led use of sensor-guided pump therapy in type 1 diabetes: a randomised controlled trial. Diabetologia 2009;52:1250-1257.

7. Raccah D, Sulmont V, Reznik Y, et al.: Incremental value of continuous glucose monitoring when starting pump therapy in patients with poorly controlled type 1 diabetes: the RealTrend study. Diabetes Care 2009;32:2245-2250.

8. Bergenstal RM, Tamborlane WV, Ahmann A, et al.: Effectiveness of sensor-augmented insulin-pump therapy in type 1 diabetes. N Engl J Med 2010;363:311-320.

9. Battelino T, Liabat S, Veeze HJ, et al.: Routine use of continuous glucose monitoring in 10,501 people with diabetes mellitus. Diabet Med 2015;32:1568-1574.

10. Nørgaard K, Scaramuzza A, Bratina N, et al.: Routine sensor-augmented pump therapy in type 1 diabetes: the INTERPRET study. Diabetes Technol Ther 2013;15:273280.

11. Boland E, Monsod T, Delucia M, et al.: Limitations of conventional methods of self-monitoring of blood glucose: lessons learned from 3 days of continuous glucose sensing in pediatric patients with type 1 diabetes. Diabetes Care 2001;24:1858-1862.

12. Bode BW, Schwartz S, Stubbs HA, Block JE: Glycemic characteristics in continuously monitored patients with type 1 and type 2 diabetes: normative values. Diabetes Care 2005;28:2361-2366.

13. Pickup JC, Freeman SC, Sutton AJ: Glycaemic control in T1DM during real time CGM compared with SMBG: metaanalysis of randomised controlled trials using individual patient data. BMJ 2011;343:d3805.

14. Juvenile Diabetes Research Foundation Continuous Glucose Monitoring Study Group, Beck RW, Buckingham B, et al.: Factors predictive of use and of benefit from continuous glucose monitoring in type 1 diabetes. Diabetes Care 2009;32: 1947-1953.

15. Anderson J, Attvall S, Sternemalm L, et al.: Effect on glycemic control by short- and long-term use of continuous glucose monitoring in clinical practice. J Diabetes Sci Technol 2011;5:1472-1479.

16. Klonoff DC, Buckingham B, Christiansen JS, et al.: Continuous glucose monitoring: an Endocrine Society Clinical Practice Guideline. J Clin Endocrinol Metab 2011;96:29682979.

17. Phillip M, Danne T, Shalitin S, et al.: Use of continuous glucose monitoring in children and adolescents. Pediatr Diabetes 2012;13:215-228.
18. American Diabetes Association: 6. Glycemic targets. Diabetes Care 2017;40(Suppl. 1):S48-S56.

19. UK National Institute for Health and Care Excellence: Integrated sensor-augmented pump therapy systems for managing blood glucose levels in type 1 diabetes (the MiniMed Paradigm Veo system and the Vibe and G4 PLATINUM CGM system). February 2016. www.nice.org.uk/guidance/ dg21 (accessed June 15, 2017).

20. National Diabetes Services Scheme, Diabetes Australia: Continuous glucose monitoring. www.ndss.com.au/cgm (accessed June 15, 2017).

21. Cohen O, Korner A, Chlup R, et al.: Improved glycemic control through continuous glucose sensor-augmented insulin pump therapy: prospective results from a community and academic practice patient registry. J Diabetes Sci Technol 2009;3:804-811.

22. Tanenbaum ML, Hanes SJ, Miller KM, et al.: Diabetes device use in adults with type 1 diabetes: barriers to uptake and potential intervention targets. Diabetes Care 2017;40:181-187.

23. Beck RW, Riddlesworth T, Ruedy K, et al.: Effect of continuous glucose monitoring on glycemic control in adults with type 1 diabetes using insulin injections: the DIAMOND randomized clinical trial. JAMA 2017;317:371-378.

24. Lind M, Polonsky W, Hirsch IB, et al.: Continuous glucose monitoring vs conventional therapy for glycemic control in adults with type 1 diabetes treated with multiple daily insulin injections: the GOLD randomized clinical trial. JAMA 2017;317:379-387.

25. Heinemann L, Deiss D, Hermanns N, et al.: HypoDE: research design and methods of a randomized controlled study evaluating the impact of real-time CGM usage on the frequency of CGM glucose values $<55 \mathrm{mg} / \mathrm{dl}$ in patients with type 1 diabetes and problematic hypoglycemia treated with multiple daily injections. J Diabetes Sci Technol 2015;9:651-662.

26. Dexcom G5 Wholesale Acquisition Cost (WAC), published by First Databank, effective March 1, 2017. http://www .fdbhealth.com/ (accessed May 2, 2017).

27. Dexcom Germany GmbH: www.nintamed.eu (accessed May 5, 2017).

28. Price after excluding VAT, Blekinge County Council report. Sweden.

29. One Diabetes Foundation: April 17, 2016. www.eendiabetes. nl/Hardware/overzicht-beschikbare-sensoren-1 (accessed June 15, 2017).

30. Tucker ME: Medicare spells out CGM coverage for diabetes. March 24, 2017. www.medscape.com/viewarticle/ 877747 (accessed May 4, 2017).

31. McQueen RB, Ellis SL, Maahs DM, et al.: Frequency of CGM use and change in $\mathrm{HbA}_{1 \mathrm{c}}$ for adults with type 1 diabetes in a clinical practice setting. Endocr Pract 2014;20:1007-1015.

32. Picard S, Hanaire H, Baillot-Rudoni S, et al.: Evaluation of the adherence to continuous glucose monitoring in the management of type 1 diabetes patients on sensor-augmented pump therapy: the SENLOCOR study. Diabetes Technol Ther 2016; 18:127-135.

33. Wong JC, Foster NC, Maahs DM, et al.: Real-time continuous glucose monitoring among participants in the T1D Exchange Clinic Registry. Diabetes Care 2014;37:2702-2709.

34. T1D Exchange: Why do some people with T1D stop using a pump and CGM? April 20, 2016. https://t1dexchange.org/ pages/why-do-some-people-with-t1d-stop-using-a-pumpand-cgm (accessed May 4, 2017).

35. Rodbard D, Bailey T, Jovanovic L, et al.: Improved quality of glycemic control and reduced glycemic variability with 
use of continuous glucose monitoring. Diabetes Technol Ther 2009;11:717-723.

36. Polonsky WH, Hessler D, Ruedy KJ, et al.: The impact of continuous glucose monitoring on markers of quality of life in adults with type 1 diabetes: further findings from the DIAMOND randomized clinical trial. Diabetes Care 2017; 40:736-741.

37. Ramchandani N, Arya S, Ten S, Bhandari S: Real-life utilization of real-time continuous glucose monitoring: the complete picture. J Diabetes Sci Technol 2011;5:860-870.

38. de Bock M, Cooper M, Retterath A, et al.: Continuous glucose monitoring adherence: lessons from a clinical trial to predict outpatient behavior. J Diabetes Sci Technol 2016; 10:627-632.
39. Mastrototaro J, Welsh JB, Lee S: Practical considerations in the use of real-time continuous glucose monitoring alerts. J Diabetes Sci Technol 2010;4:733-739.

40. Heinemann L, Franc S, Phillip M, et al.: Reimbursement for continuous glucose monitoring: a European view. J Diabetes Sci Technol 2012;6:1498-1502.

Address correspondence to: Shengsheng $\mathrm{Yu}, \mathrm{PhD}$ Abbott Diabetes Care 1420 Harbor Bay Pkwy Alameda, CA 94502

E-mail: shengsheng.yu@abbott.com 